\title{
LOCALLY NOETHERIAN COMMUTATIVE RINGS
}

\author{
BY \\ WILLIAM HEINZER AND JACK OHM( $\left.{ }^{1}\right)$
}

\begin{abstract}
This paper centers around the theorem that a commutative ring $R$ is noetherian if every $R_{P}, P$ prime, is noetherian and every finitely generated ideal of $R$ has only finitely many weak-Bourbaki associated primes. A more precise local version of this theorem is also given, and examples are presented to show that the assumption on the weak-Bourbaki primes cannot be deleted nor replaced by the assumption that $\operatorname{Spec}(R)$ is noetherian. Moreover, an alternative statement of the theorem using a variation of the weak-Bourbaki associated primes is investigated. The proof of the theorem involves a knowledge of the behavior of associated primes of an ideal under quotient ring extension, and the paper concludes with some remarks on this behavior in the more general setting of flat ring extensions.
\end{abstract}

Our terminology is that of Zariski-Samuel [17] and Bourbaki [3]. We use $R$ to denote a commutative ring with identity and $A$ to denote an ideal of $R$. (All rings of this paper are assumed to be commutative with identity.) By "ideal" we shall always mean an ideal $\neq R$. A prime ideal $P$ of $R$ is called an MPD (minimal prime divisor) of $A$ if $P$ is minimal among the prime ideals containing $A$. $A$ is said to be a radical ideal if $A=\sqrt{ } A$. If $S$ and $T$ are sets, we use $S \backslash T$ to denote $\{s \in S \mid s \notin T\}$; and $\subset$ denotes containment and $<$ strict containment.

1. The main theorem. We shall discuss in detail in $\$ 3$ the concept of associated primes of an ideal, but for the present it will suffice to give the following definition. A prime ideal $P$ of $R$ is called a $B_{w}$-prime of $A$ (weak-Bourbaki associated prime, [3b, p. 165, Exercise 17]) if there exists $x \in R$ such that $P$ is an MPD of $A: x$.

1.1. Lemma. If $A$ has a unique $B_{w}$-prime $P$, then $A$ is $P$-primary (and conversely).

Proof. Suppose $x y \in A, x \notin P$. If $y \notin A$, then $A: y<R$. But $x \in A: y$ implies $A: y \notin P$; so if $Q$ is an MPD of $A: y$, then $Q$ is a $\mathrm{B}_{\text {w }}$-prime of $A$ different from $P$. Thus, $y \in A$.

1.2. Proposition. Let $R^{\prime}$ be a quotient ring of $R$ (with respect to a multiplicative system), let $A$ be an ideal of $R$ and $A^{\prime}=A R^{\prime}$, and let $P$ be a prime ideal of $R$ such that $P^{\prime}=P R^{\prime} \neq R^{\prime}$. Then $P$ is a $B_{w}$-prime of $A$ if and only if $P^{\prime}$ is a $B_{w}$-prime of $A^{\prime}$.

Presented to the Society, January 24, 1969 under the title Noetherian and nonnoetherian commutative rings; received by the editors October 21, 1968 and, in revised form, August 7, 1970.

AMS 1970 subject classifications. Primary 13E05, 13 A15.

Key words and phrases. Associated prime ideal, locally noetherian ring, noetherian spectrum, flat ring extension.

( $\left.{ }^{1}\right)$ The authors received partial support from the National Science Foundation during the writing of this paper. During completion of the paper the first named author was supported as an NRC-ONR Research Associate. 
Proof. $\Rightarrow . P$ is an MPD of $A: x$ for some $x \in R$. Since $R^{\prime}$ is a quotient ring of $R$, $(A: x) R^{\prime}=A R^{\prime}: x$; and there exists a $1-1$ correspondence between primes of $R^{\prime}$ and primes of $R$ which do not blow up, effected by extension and contraction. Therefore $P^{\prime}$ is an MPD of $A R^{\prime}: x$.

$\Leftarrow P^{\prime}$ is a $\mathrm{B}_{\mathrm{w}}$-prime of $A^{\prime}$ implies there exists $y \in R^{\prime}$ such that $P^{\prime}$ is an MPD of $A^{\prime}: y$. Then $P^{\prime} R_{P^{\prime}}^{\prime}$ is an MPD of $\left(A^{\prime}: y\right) R_{P^{\prime}}^{\prime}=A^{\prime} R_{P^{\prime}}^{\prime}: y$. But $R_{P^{\prime}}^{\prime}$ is canonically isomorphic to $R_{P}$, so if $\varphi$ is the canonical homomorphism of $R$ into $R_{P^{\prime}}^{\prime}$, then there exists $s \in R \mid P$ and $r \in R$ such that $\varphi(s)$ is a unit in $R_{P^{\prime}}^{\prime}$ and $\varphi(s) y=\varphi(r)$. Therefore $A^{\prime} R_{P^{\prime}}^{\prime}: y=A^{\prime} R_{P^{\prime}}^{\prime}: \varphi(s) y=(A: r) R_{P^{\prime}}^{\prime}$, the last equality being a consequence of the fact that $R_{P^{\prime}}^{\prime}$ is a quotient ring of $R$. It follows that $P R_{P}$ is an MPD of $(A: r) R_{P}$, and hence $P$ is an MPD of $A: r$. Q.E.D.

The above proposition is an exercise in [3b, p. 166]. We include a proof because we are interested in $\$ 4$ in the degree to which Proposition 1.2 remains true when we replace the hypothesis that $R^{\prime}$ is a quotient ring of $R$ by the weaker assumption that $R^{\prime}$ is merely a flat $R$-algebra.

In the proofs of the next theorem and its corollary we use the following lemma:

LEMMA. Let $T_{i}=\left\{P_{i j}\right\}_{j=1}^{n_{1}}, i=1,2, \ldots$, be a collection of finite sets ( possibly empty), and let $T=\bigcup T_{i}$. Suppose that $T$ is partially ordered with respect to a relation $\leqq$ and that $T$ with respect to this relation satisfies the ascending chain condition and has the further property that if, $P_{i+1 j} \in T_{i+1}$, then there exists $P_{i k} \in T_{i}$ such that $P_{i+1 j}>P_{i k}$. Then there exists a natural number $n$ such that $T_{i}$ is empty for all $i \geqq n$.

Proof. Suppose that for each natural number $n$ there exists $i \geqq n$ such that $T_{i}$ is nonempty. It follows from our hypotheses that $T_{i}$ is nonempty for all $i$. Let $T_{1}^{n}$ $=\left\{P_{1 j_{1}} \mid\right.$ there exists a chain $\left.P_{1 j_{1}}<P_{2 j_{2}}<\cdots<P_{n j_{n}}\right\}$. Since $T_{n}$ is nonempty, it follows that $T_{1}^{n}$ is also nonempty. Moreover, $T_{1}^{n} \supset T_{1}^{m}$ for $m \geqq n$; and $T_{1}^{n}$ is finite. Therefore $\bigcap_{n=1}^{\infty} T_{1}^{n}$ is nonempty. Choose $P_{1} \in \bigcap_{n=1}^{\infty} T_{1}^{n}$.

Now, for each $n \geqq 2$, let $T_{2}^{n}=\left\{P_{2 j_{2}} \mid\right.$ there exists a chain $\left.P_{1}<P_{2 j_{2}}<\cdots<P_{n j_{n}}\right\}$. Since $P_{1} \in T_{1}^{n}, T_{2}^{n}$ is nonempty. Also, $T_{2}^{n} \supset T_{2}^{m}$ for $m \geqq n$; and $T_{2}^{n}$ is finite. Therefore $\bigcap_{n=2}^{\infty} T_{2}^{n}$ is nonempty. Choose $P_{2} \in \bigcap_{n=2}^{\infty} T_{2}^{n}$.

Thus, proceeding inductively, we construct a chain $P_{1}<P_{2}<\cdots$, which contradicts the ascending chain condition.

1.3. THEOREM. Let $P$ be a prime ideal of $R$, and suppose the following hold:

(i) $P R_{M}$ is finitely generated for every maximal ideal $M \supset P$ (and hence also for every prime ideal containing $P$ ),

(ii) there exists a finitely generated ideal $B_{0}$ such that $\sqrt{ } B_{0}=P$ and such that for every finitely generated ideal $B$ with $B_{0} \subset B \subset P, B$ has only finitely many $B_{w}$-primes,

(iii) the set of $B_{w}$-primes of the ideals $B$ of (ii) satisfies the a.c.c. (ascending chain condition).

Then $P$ is finitely generated.

Proof. Let $B$ be a finitely generated ideal of $R$ such that $B_{0} \subset B \subset P$ and such that $B R_{P}=P R_{P}$. By (ii), $B$ has only finitely many $B_{\mathrm{w}}$-primes different from $P$, say 
$P_{1}, \ldots, P_{n}$. Then $\sqrt{ } B=P$ implies $P \subset P_{i}$. Since the $P_{i}$ are finite in number and each $P R_{P_{1}}$ is finitely generated (by (i)), there exists a finitely generated ideal $A$ such that $B \subset A \subset P$ and $A R_{P_{i}}=P R_{P_{i}}, i=1, \ldots, n$. Note that each $\mathrm{B}_{\mathrm{w}}$-prime of $A$ contains $P=\sqrt{ } A$.

Claim. If $Q_{1}, \ldots, Q_{m}$ is the set of $\mathrm{B}_{\mathrm{w}}$-primes of $A$ different from $P$, then each $Q_{i}$ properly contains some $P_{j}$. To see this, it suffices to show that if $Q$ is a prime ideal of $R$ which contains $P$ but does not properly contain some $P_{j}$, then $A R_{Q}=P R_{Q}$; for $A R_{Q_{i}}$ has $Q_{i} R_{Q_{i}}$ as a $\mathrm{B}_{\mathrm{w}}$-prime by Proposition 1.2, and hence $A R_{Q_{i}} \neq P R_{Q_{i}}$. If $Q=P_{j}$ for some $j$, then by the choice of $A, A R_{Q}=P R_{Q}$. On the other hand, if $Q \neq P$, for all $j$, then $B R_{Q}$ has $P R_{Q}$ as its unique $\mathrm{B}_{\mathrm{w}}$-prime by 1.2. But then $B R_{Q}$ is $P R_{Q}$-primary by Lemma 1.1. Since $R_{P}$ is a quotient ring of $R_{Q}$ and $B R_{P}=P R_{P}$, we therefore conclude that $B R_{Q}=P R_{Q}$, and a fortiori $A R_{Q}=P R_{Q}$.

We proceed to define inductively a chain $\left\{A_{i}\right\}$ of finitely generated ideals containing $B_{0}$ and contained in $P$ and such that $A_{i} R_{P}=P R_{P}$. We take $A_{0}$ to be the ideal generated by the ideal $B_{0}$ of (ii) and a finite set of elements of $R$ which generate $P R_{P}$ (by (i) such a set exists). Then $B_{0} \subset A_{0} \subset P$ and $A_{0} R_{P}=P R_{P}$. If now $N_{1}, \ldots, N_{r}$ are the $\mathrm{B}_{\mathrm{w}}$-primes of $A_{n}$ distinct from $P$, then we choose $A_{n+1}$ to be a finitely generated ideal such that $A_{n} \subset A_{n+1} \subset P$ and $A_{n+1} R_{N_{t}}=P R_{N_{t}}, i=1, \ldots, r$. By our previous observations and the a.c.c. on the $\mathrm{B}_{\mathrm{w}}$-primes of the $\left\{A_{i}\right\}$, we can apply the above lemma to conclude that there exists $m$ such that $A_{m}$ has $P$ as its unique $\mathrm{B}_{\mathrm{w}^{-}}$ prime. Then $A_{m}$ is $P$-primary by Lemma 1.1. Since $A_{m} R_{P}=P R_{P}$, we therefore conclude that $A_{m}=P$. Thus, $P$ is finitely generated. Q.E.D.

Note that the converse to Theorem 1.3 is trivially true. The following corollary gives a criterion for $R$ to be noetherian when every localization of $R$ is. In $\S \S 2$ and 3 we shall justify by examples the hypotheses used here.

\subsection{Corollary. If every $R_{P}, P$ a prime ideal of $R$, is noetherian and every finitely} generated ideal of $R$ has only finitely many $B_{w}$-primes, then $R$ is noetherian.

Proof. By Cohen's theorem, it is sufficient to check that every prime ideal $P$ of $R$ is finitely generated. Any chain of primes is contained in a maximal prime $M$, so $R_{M}$ is noetherian implies the chain must be finite; thus, (iii) of Theorem 1.3 is satisfied. We must yet check that $P=\sqrt{ } B_{0}$ for some finitely generated ideal $B_{0}$. Since $P R_{P}$ is finitely generated, there exists a finitely generated ideal $A$ of $R$ such that $A R_{P}=P R_{P}$. Since $A$ has only finitely many $\mathrm{B}_{\mathrm{w}}$-primes by hypothesis, $A$ has a fortiori only finitely many MPD's, one of them being $P$. Therefore we can enlarge $A$ to a finitely generated ideal $A_{1}$ such that $A \subset A_{1} \subset P$ and such that no MPD of $A_{1}$ different from $P$ is an MPD of $A$. We similarly enlarge $A_{1}$ and thus construct a chain $A \subset A_{1} \subset A_{2} \subset \ldots \subset P$ such that no MPD of $A_{i+1}$ different from $P$ is an MPD of $A_{i}$. By applying the above lemma to the MPD'S different from $P$ of the $A_{i}$, we conclude that there exists an $n$ such that $P$ is the only MPD of $A_{n}$. Therefore $A_{n}=B_{0}$ is the required ideal. Q.E.D. 
2. Two examples. We shall give in this section two examples to show that some obvious conjectures in connection with Corollary 1.4 must be ruled out. First, the most obvious conjecture is that every $R_{P}$ is noetherian implies $R$ is noetherian. The customary counterexample is due to Nakano [13, p. 426] and is quite involved. (See also [2, §4] where a generalized form is worked out.) It is possible to give a simple example, which we do in 2.2 .

Thus, the assumption in 1.4 that every finitely generated ideal of $R$ has only finitely many $B_{w}$-primes, or some alternative, is certainly necessary for the validity of the corollary. A pleasant alternative would be the assumption that $\operatorname{Spec}(R)$ is noetherian, i.e. that radical ideals of $R$ have a.c.c. Some equivalent statements are the following [15]:

(i) $\operatorname{Spec}(R)$ is noetherian.

(ii) For any ideal $A$ of $R$, there exists a finitely generated ideal $A_{0}$ such that $\sqrt{ } A=\sqrt{ } A_{0}$.

(iii) For any prime ideal $P$ of $R$, there exists a finitely generated ideal $P_{0}$ such that $P=\sqrt{ } P_{0}$.

(iv) Every ideal of $R$ has only finitely many MPD's and $R$ has a.c.c. on prime ideals.

Our example 2.3 is considerably more complicated than 2.2 and is a finitedimensional domain $R$ which satisfies (i)-(iv) and has every $R_{P}$ noetherian but is not itself noetherian. Thus, this condition is not suitable either. Incidentally, the example also shows then that the assumption in Theorem 1.3 that every finitely generated ideal $B$ such that $B_{0} \subset B \subset P$ have only finitely many $\mathrm{B}_{\mathrm{w}}$-primes cannot be omitted.

2.2. Example of a domain $D$ such that every $D_{P}$ is a noetherian valuation ring but $D$ is not itself noetherian.

Since this example uses the technique of constructing a domain by means of an appropriate lattice ordered group, we shall review first a few of the facts related to this construction. If $D$ is a domain with quotient field $K$, let $U(D)$ denote the group of units of $D$, and let $*$ denote nonzero elements. Let $\varphi: K^{*} \rightarrow K^{*} / U(D)=G$ be the canonical homomorphism of the (multiplicative) group $K^{*}$ onto the group $G$. It is customary to switch to additive notation for $G$, and we do so. An ordering is defined on $G$ by taking $G^{+}=\varphi\left(D^{*}\right)$, and the resulting ordered group is called the group of divisibility of $D$ (where here $G^{+}=\{g \in G \mid g \geqq 0\}$ ). We are particularly interested in the case when $G$ is lattice ordered, since we then have

THEOREM (JAFFARD [8, p. 78]). If $G$ is a lattice ordered group, then there exists a domain $D$ having $G$ as group of divisibility. We henceforth assume that $G$ denotes a lattice ordered group (although much of what follows can be done in greater generality; see [14]).

We now carry over the ideal-theoretic concepts from $D$ to $G$. A segment of a lattice ordered group $G$ is a subset $A \neq G^{+}$of $G^{+}$such that $a \in A$ and $b \geqq a$ implies 
$b \in A$, and $a, b \in A$ implies inf $\{a, b\} \in A$. $A$ is called a prime segment if $a, b \in G^{+} \backslash A$ implies $a+b \notin A$. $A$ is a principal segment if there exists $a \in A$ such that $A=\left\{b \in G^{+} \mid b \geqq a\right\} . G$ is noetherian if the a.c.c. holds for the segments. $\operatorname{dim} G$ (or rank $G)=n$ if there exists a chain $P_{1}<P_{2}<\cdots<P_{n}$ of $n$ prime segments and no such longer chain, etc. Finally, let us sketch yet how the concept of localization at a prime carries over to $G$ (see [8, p. 36]). If $P$ is a prime segment of $G$, let $H$ be the subgroup of $G$ generated by $G^{+} \backslash P$. Let $\varphi_{P}$ be the canonical homomorphism $G \rightarrow G / H=G_{P}$, and define an order on $G_{P}$ by taking $G_{P}^{+}=\varphi_{P}\left(G^{+}\right) . G_{P}$ is the localization of $G$ at $P$. If $P^{\prime}=\varphi^{-1}(P) \cup 0$ then $P^{\prime}$ is a prime ideal of $D$; and $G_{P}$ is then the group of divisibility of $D_{P^{\prime}}$, with $\varphi_{P} \cdot \varphi: K^{*} \rightarrow G_{P}$ being the associated group of divisibility homomorphism. Note in particular that when $G_{P}$ is totally ordered, then $\varphi_{P} \cdot \varphi$ is a valuation having ring $D_{P^{\prime}}$.

We conclude this summary with a few elementary observations about the relationship between $D$ and $G$. If $A$ is any segment (prime segment) of $G$, then $\varphi^{-1}(A) \cup\{0\}$ is an ideal (prime ideal) of $D$. Conversely, if one uses the $K$ and $\varphi$ given by the proof of Jaffard's theorem, it can be seen that for any ideal $A \neq(0)$ of $D$, $\varphi\left(A^{*}\right)$ is a segment of $G$, and $\varphi^{-1}\left(\varphi\left(A^{*}\right)\right)=A^{*}$. Thus, if one uses Jaffard's construction, there exists a 1-1 correspondence between nonzero ideals of $D$ and segments of $G$; and this correspondence preserves primeness and most of the other idealtheoretic concepts. It can furthermore be seen [7, p. 1370] that the $D$ given by Jaffard's construction is actually a Bezout domain (i.e. finitely generated ideals are principal).

To construct example 2.2, it is sufficient then to give a lattice ordered group $G$ such that for every prime segment $P$ of $G$, the group $G_{P}$ is order isomorphic to $Z$, where $Z=$ additive group of integers with the usual order. The idea for the example comes from a similar example given by Underwood in [16, Example 3.10].

Let $N=\{1,2,3, \ldots\}, Z^{+}=\{0,1,2,3, \ldots\}$. Let $G=$ group of all functions from $N$ to $Z$ such that $f(i)$ is constant for all $i$ greater than some integer, i.e. $G=\left\{f \in Z^{N} \mid f(N)\right.$ is finite $\}$. $G$ is a lattice ordered group under the ordering $f \leqq g$ if and only if $f(i) \leqq g(i)$ for all $i \in N$. Let

$$
\begin{aligned}
P_{i} & =\{f \in G \mid f(i)>0\}, \quad i=1,2, \ldots, \\
P_{\infty} & =\{f \in G \mid \text { there exists } n \text { such that } f(i)>0 \text { for all } i>n\} .
\end{aligned}
$$

$P_{i}, P_{\infty}$ are clearly prime segments of $G$.

Claim. These are the only prime segments. For suppose $Q$ is a prime segment $\notin P_{i}, P_{\infty}, i=1,2, \ldots$ Since $Q \nsubseteq P_{\infty}$, there exists $f_{\infty} \in Q$ such that $f_{\infty}(i)=0$ for all $i>$ some integer $n$. Also, $Q \nsubseteq P_{1}, \ldots, P_{n}$ implies there exists $f_{j} \in Q$ such that $f_{j}(j)=0$, $j=1, \ldots, n$. Therefore, $0=\inf \left\{f_{1}, \ldots, f_{n}, f_{\infty}\right\} \in Q$, a contradiction. Thus, $P_{i}, P_{\infty}$ are the only maximal segments of $G$. Now consider the maps $\varphi_{i}: G \rightarrow Z, i=1,2$, $\ldots, \infty$, defined by

$$
\begin{aligned}
\varphi_{j}(f) & =f(j), \quad j=1,2, \ldots, \\
\varphi_{\infty}(f) & =f(n+1),
\end{aligned}
$$


where $n+1$ is chosen such that $f$ is constant for $i \geqq n+1$. The $\varphi_{i}$ are lattice homomorphisms of $G$ onto $Z$. Since $Z$ has the unique prime segment $Z{ }^{+} \mid 0$, it follows from the usual correspondence between prime segments under a lattice homomorphism that $P_{i}$ contains no prime segment properly. Thus, $P_{i}, i=1, \ldots, \infty$, are the only prime segments of $G$; and $G_{P_{t}}$ is order isomorphic to $Z$. Finally, $G$ is not noetherian since $f=1$ has infinitely many MPD's. It is also interesting to note that each $P_{i}, i<\infty$, is principal but $P_{\infty}$ is not.

2.3. Example of a (2-dim) domain $R$ such that $R_{P}$ is noetherian for every prime ideal $P$ of $R$ and $\operatorname{Spec}(R)$ is noetherian, but $R$ is not noetherian. (By Corollary 1.4, a 1-dim example of this type cannot exist.) It follows from the usual theorems on integral extensions that if $R \subset R^{\prime}$ and $R^{\prime}$ is integral over $R$, then $R^{\prime}$ satisfies (iv) of 2.1 implies $R$ does also; so it is sufficient to exhibit a nonnoetherian domain $R$ such that

(a) $R_{P}$ is noetherian for every prime $P$ of $R$,

(b) there exists a noetherian domain $R^{\prime}$ such that $R^{\prime}$ is integral over $R$.

Let $k$ be a field of characteristic 0 and $x_{1}, x_{2}, \ldots, y, z$ be indeterminates over $k$, let $K=k\left(x_{1}, x_{2}, \ldots\right)$, and let $J=K[y, z] . P_{i}=(y, z-i), i=1,2, \ldots$, are prime ideals of $J$; and $J_{P_{i}}=R_{i}^{\prime}$ are 2-dim local domains of the form $R_{i}^{\prime}=K+M_{i}$, where $M_{i}$ =maximal ideal of $R_{i}^{\prime}$. Consider the subring $R_{i}=K_{i}+M_{i}$ of $R_{i}^{\prime}$, where $K_{i}=k\left(x_{1}, x_{2}, \ldots, x_{i}^{i}, \ldots\right)$ is the subfield of $K$ generated by $\left\{x_{j}\right\}_{j \neq i}$ and $x_{i}^{i}$ over $k$. Note that $R_{i}^{\prime}$ is a finite integral extension of $R_{i}$ and hence that $R_{i}$ is a 2-dim local domain. (That $R_{i}$ is noetherian can easily be checked directly, or else apply Eakin's theorem [4] or [11].) Let $R=\bigcap R_{i}$ and $R^{\prime}=\bigcap R_{i}^{\prime}=\bigcap J_{P_{i}} \cdot R^{\prime}$ is then a Krull ring whose essential valuation rings are the localizations of $J$ at those minimal primes which are contained in some $P_{i}$. Since the minimal primes of $J$ are principal, this set can also be described as the set of minimal primes of $J$ which are contained in $\bigcup P_{i}$. Therefore by $[12$, p. $116,33.5], R^{\prime}=J_{S}$, where $S=J \backslash \cup P_{i}$.

We next note that $\left\{P_{i}\right\}$ is exactly the set of primes of $J$ which are maximal with respect to not meeting $S$. For, if $Q \neq 0$ is a prime of $J$ such that $Q \subset \cup P_{i}$, then $Q$ has height 1 or 2 . Since $J$ is a UFD, $Q$ has height 1 implies $Q$ is principal; and then $Q \subset P_{i}$ for some $i$. If height $Q=2$, then $Q$ is maximal in $J$; so $Q+(y) \subset \cup P_{i}$ implies $y \in Q$. But $Q$ is principal $\bmod (y)$, so $Q=(y, f), f \in K[z]$. Then $f \in \bigcup P_{i}$ implies $f \in P_{i}$ for some $i$ : so $Q \subset P_{i}$. It now follows that $\left\{P_{i} J_{S}\right\}$ is exactly the set of maximal ideals of $J_{S}=R^{\prime}$.

Now let us check that $R^{\prime}$ is integral over $R . r^{\prime} \in R^{\prime}$ implies $r^{\prime} \in k\left(x_{1}, \ldots, x_{n}\right)(y, z)$ $=L_{n}$, for some $n . r^{\prime} \in R_{i}^{\prime} \cap L_{n}$ implies $r^{\prime}=a_{i}+b_{i}, a_{i} \in k\left(x_{1}, \ldots, x_{n}\right), b_{i} \in M_{i}$. Since $a_{1}, \ldots, a_{n}$ are integral over $k\left(x_{1}, x_{2}^{2}, \ldots, x_{n}^{n}\right)$, there exists a monic polynomial $f(T) \in k\left(x_{1}, x_{2}^{2}, \ldots, x_{n}^{n}\right)[T]$ such that $f\left(a_{i}\right)=0, i=1, \ldots, n$. Therefore $f\left(r^{\prime}\right) \in M_{i}$ $\subset R_{i}, i=1, \ldots, n$; so $f\left(r^{\prime}\right) \in \bigcap R_{i}=R$. Thus, $r^{\prime}$ is integral over $R$.

Let us denote the prime $P_{i} J_{S}$ of $R^{\prime}$ by $N_{i}^{\prime}$. As we have observed, $\left\{N_{i}^{\prime}\right\}$ is exactly the set of maximal ideals of $R^{\prime}$. Since $R^{\prime}$ is integral over $R$, it follows that $\left\{N_{i}\right\}$, where $N_{i}=N_{i}^{\prime} \cap R$, is exactly the set of maximal ideals of $R$. To show each $R_{P}$ is 
noetherian, it suffices to show then that each $R_{N_{t}}$ is noetherian. We shall, in fact, show that $R_{N_{i}}=R_{i} . R_{N_{i}} \subset R_{i}$ is clear since $N_{i}$ is contained in the maximal ideal $M_{i}$ of $R_{i}$. Conversely, if $a \in R_{i}$, we must exhibit an $r \in R \backslash N_{i}$ such that $r a \in R$. Since $R^{\prime}=J_{S}$ and $R_{i}^{\prime}=J_{P_{i}}$ and $N_{i}^{\prime}=P_{i} J_{S}$, it follows that $a \in R_{i}^{\prime}=R_{N_{i}^{\prime}}^{\prime}$. Therefore there exists $r^{\prime} \in R^{\prime} \backslash N_{i}^{\prime}$ such that $r^{\prime} a \in R^{\prime}$. If $L_{n}=k\left(x_{1}, \ldots, x_{n}, y, z\right)$, then $r^{\prime} a$ and $r^{\prime}$ are in $L_{n}$ for some $n$; so $r^{\prime} a$ and $r^{\prime}$ are in $R_{j}, j>n$. We also have $r^{\prime}=b+m, b \in K \cap L_{n}$, $m \in M_{i}$; and since $r^{\prime}$ is a unit of $R_{i}^{\prime}$, we see that $b \neq 0$. Hence there is a monic polynomial $g(T) \in\left(K_{i} \cap L_{n}\right)[T]$ such that $g(b)$ is a nonzero element of $K_{i}$. It follows that $g\left(r^{\prime}\right)$ is a unit of $R_{i}$. Also, $a \cdot g\left(r^{\prime}\right) \in R^{\prime}$; so if $r=\pi(z-j) g\left(r^{\prime}\right), j=1, \ldots, n, j \neq i$, then $r \in R \backslash N_{i}$ and $r a \in R$. Thus, $R_{N_{i}}=R_{i}$ and each localization of $R$ is noetherian.

Finally, we observe that $R$ is not itself noetherian. For, if $i \geqq 2$, then $x_{i} y \notin y R$ but $(z-i) x_{i} y \in y R$. Since $R^{\prime}$ is integral over $R$ and $N_{i}^{\prime}$ is the only prime of $R^{\prime}$ which contains both $y$ and $z-i$, it follows that $N_{i}=\sqrt{ }\left(y R: x_{i} y\right), i \geqq 2$. Thus, $y R$ is an ideal of $R$ with an infinite number of $B_{w}$-primes (in fact, an infinite number of ZS-primes; see $\S 3$ ), so $R$ is not noetherian.

3. Associated prime ideals. We devote this section to an examination of some alternative hypotheses in Theorem 1.3 and Corollary 1.4. Let us begin by considering some possible alternatives to the $\mathrm{B}_{\mathrm{w}}$-primes we employ there. The following four definitions of associated primes of an ideal $A$ can be found, either as definitions or as characterizations, in [3b, p. 131], [17, Vol. 1, p. 211], [3b, p. 165, Exercise 17], and $[12, \S 7]$, respectively.

3.1. Definitions. (B) $P$ is an associated prime of $A$ in the Bourbaki sense if $P=A: x$ for some $x \in R$.

(ZS) $P$ is an associated prime of $A$ in the Zariski-Samuel sense if there exists $x \in R$ such that $P=\sqrt{ }(A: x)$.

(B) $P$ is an associated prime of $A$ in the weak-Bourbaki sense if there exists $x \in R$ such that $P$ is an MPD (minimal prime divisor) of $A: x$.

(N) $P$ is an associated prime of $A$ in the Nagata sense if there exists a multiplicative system $S$ of $R$ such that $S \cap A=\varnothing$ and such that $P R_{S} \supset A R_{S}$ and $P R_{S}$ is maximal with respect to being contained in the set of zero-divisors $\bmod A R_{S}$.

When $R$ is noetherian, then the associated primes in any of the above senses coincide with the usual associated primes of $A$. In general, $P$ satisfies $(\mathrm{B}) \Rightarrow P$ satisfies $(\mathrm{ZS}) \Rightarrow P$ satisfies $\left(\mathrm{B}_{\mathrm{w}}\right) \Rightarrow P$ satisfies $(\mathrm{N})$, and none of these implications is reversible. See [16] for details $\left({ }^{2}\right)$.

(B) and (ZS) seem to be too restrictive to be of much use in the nonnoetherian case; for example, an ideal may have no ZS-primes. On the other hand, at least every MPD of an ideal must be a $B_{w}$-prime. Moreover, as we have shown in Proposition 1.2, the $\mathrm{B}_{\mathrm{w}}$-primes localize well.

It is conceivable that replacing " $\mathrm{B}_{\mathrm{w}}$-primes" by " $\mathrm{ZS}$-primes" in Corollary 1.4 would yield a better result. However, it can be seen that Nakano's example [13,

$\left.{ }^{2}\right)$ The ZS-primes defined by Underwood in [16] are slightly different from our ZS-primes. 
p. 426] gives a domain $D$ such that every $D_{P}, P$ prime, is a noetherian valuation ring and yet every finitely generated ideal of $D$ has no ZS-primes; so 1.4 is, in fact, false with this replacement. (Such a domain can also be constructed by using Jaffard's theorem as in 2.2 but we shall omit the details. Note that 2.2 does not itself yield the required example since it is easily seen that there exist principal ideals of that example which have infinitely many ZS-primes.) We shall show in Corollary 3.4 though that if a mild condition is added to the hypothesis of 1.4, then such a change to ZS-primes can be successfully made. We first examine how to reformulate Theorem 1.3 and Corollary 1.4 in terms of ZS-primes.

3.2. Lemma. Let $A$ be an ideal of $R$. If $\{\sqrt{ }(A: x)\}, x \in R$, has a.c.c., then every $B_{w}$-prime of $A$ is also a ZS-prime.

Proof. Suppose $A$ has a $\mathrm{B}_{\mathrm{w}}$-prime $P$ which is not a ZS-prime. Among the ideals of the form $\sqrt{ }(A: x)$ which have $P$ as an MPD, choose a maximal such ideal $B=\sqrt{ }(A: x) . P \neq B$ since $P$ is not a ZS-prime of $A$, so there exists an MPD $Q$ of $B$ such that $Q \neq P$. Choose $y \in Q \backslash P$. $y$ is in an MPD of $B$ implies $y$ is a zero-divisor $\bmod B$ [3a, p. 94]. Therefore, there exists $z \notin B$ such that $z y \in B$. By raising to a power, we may assume $z y \in A: x$. If now $B^{\prime}=\sqrt{ }(A: x y)$, then $B \subset B^{\prime}$ and $z \in B^{\prime}$; so $B<B^{\prime}$. Moreover, $y \notin P$ implies $A: x y=(A: x): y \subset P$; so $B^{\prime} \subset P$. Thus, $B^{\prime}$ contradicts the maximality of $B$. Q.E.D.

The following theorem is our version of Theorem 1.3 in terms of ZS-primes.

3.3. THEOREM. If $P$ is a prime ideal of $R$ such that

(i) $P R_{M}$ is finitely generated for every maximal ideal $M \supset P$,

(ii) there exists a finitely generated ideal $B_{0}$ such that $\sqrt{ } B_{0}=P$ and such that for every finitely generated ideal $B$ with $B_{0} \subset B \subset P, B$ has only finitely many $Z S$-primes,

(iii) the set of ideals of the form $\sqrt{ }(B: x), B$ an ideal from (ii), $x \in R$, satisfies the a.c.c.,

then $P$ is finitely generated.

Proof. By Lemma 3.2 the ZS- and $B_{w}$-primes of an ideal $B$ of (ii) coincide. (iii) implies the a.c.c. on ZS-primes of ideals $B$ from (ii) and hence also the a.c.c. on $B_{w}$-primes of such ideals. Therefore Theorem 1.3 applies. Q.E.D.

The next corollary is the ZS-analogue of Corollary 1.4.

3.4. COROLlary. If $R$ is a ring such that

(i) every $R_{P}, P$ prime, is noetherian,

(ii) every finitely generated ideal of $R$ has only finitely many ZS-primes,

(iii) $\operatorname{Spec}(R)$ is noetherian,

then $R$ is noetherian.

Proof. Since $\operatorname{Spec}(R)$ is noetherian, $R$ has a.c.c. on radical ideals. Therefore Theorem 3.3 applies; so every prime ideal of $R$ is finitely generated, and hence by Cohen's theorem $R$ is noetherian. Q.E.D. 
We conclude this section with some further observations on the associated primes of an ideal.

3.5. Proposition. Let $A$ be an ideal of $R$. If $A$ has only finitely many $B_{w}$-primes, then $\{Z S$-primes $\}=\left\{B_{w}\right.$-primes $\}=\{N$-primes $\}$.

Proof. Since the hypothesis of 3.2 is satisfied when $A$ has only finitely many $\mathrm{B}_{\mathrm{w}}$-primes, every $\mathrm{B}_{\mathrm{w}}$-prime of $A$ is also a ZS-prime. By our previous remarks, it therefore remains to see that every $\mathrm{N}$-prime of $A$ is a $\mathrm{B}_{\mathrm{w}}$-prime. First consider the case where $P$ is a maximal $\mathrm{N}$-prime of $A$, i.e. a prime which is maximal with respect to containing $A$ and being contained in the zero-divisors $\bmod A$. Let $Q_{1}, \ldots, Q_{n}$ be the $\mathrm{B}_{\mathrm{w}}$-primes of $A$ which do not contain $P$. Choose $x \in P, \notin Q_{1}$ $\cup \cdots \cup Q_{n}$. Since $x$ is a zero-divisor $\bmod A$, there exists $y \notin A$ such that $x y \in A$. Then $x \in A: y$ implies $A: y \notin Q_{1} \cup \cdots \cup Q_{n}$; and since any MPD of $A: y$ is a $\mathrm{B}_{\mathrm{w}^{-}}$ prime of $A$, it follows that $P$ is the only MPD of $A: y$. Thus, $P$ is a $\mathrm{B}_{\mathrm{w}}$-prime of $A$ (and even a ZS-prime of $A$ ). Since $\mathrm{B}_{\mathrm{w}}$-primes localize well (1.2), and since every $\mathrm{N}$-prime extends to a maximal $\mathrm{N}$-prime in some $R_{S}$, it follows that every $\mathrm{N}$-prime of $A$ is a $\mathrm{B}_{\mathrm{w}}$-prime. Q.E.D.

Underwood proves in [16, Theorem 3.4 and Proposition 3.6] that if $A=\sqrt{ } A$, then the ZS- and B-primes coincide with the irredundant MPD's of $A$ ( $P$ is an irredundant MPD of $A$ if it cannot be deleted when $A$ is written as the intersection of all its MPD's). Nakano's example [13, p. 426] shows that an ideal $A$ may have no ZS-primes but infinitely many $B_{w}$-primes; and Underwood gives examples in [16, Examples 3.9-3.15] which show that ZS-primes and $\mathrm{B}_{\mathrm{w}}$-primes of an ideal $A$ may be present in almost any combination.

Also, in connection with Lemma 3.2 and Proposition 3.5, it is possible to have an ideal $A$ such that $\{\sqrt{ }(A: x)\}, x \in R$, satisfies the a.c.c. but $A$ still does not have a finite number of $B_{w}$-primes; this example was used in another context in [16, 3.9].

3.6. ExAMPLE. Let $R=k\left[x_{1}, x_{2}, \ldots\right]$ be a polynomial ring over the field $k$ in the indeterminates $x_{i}$, and let $A=\left(\left\{x_{i} x_{j}\right\}_{i<j}\right)$. Then $A=\bigcap P_{n}, P_{n}=\left(x_{1}, x_{2}, \ldots, x_{n-1}\right.$, $\left.x_{n+1}, \ldots\right)$; and $\left\{P_{n}\right\}$ is the set of MPD's of $A$.

If $A: r \notin P_{n}, r \in R$, then $r \in P_{n}$; so $x_{n} r \in A$, and hence $x_{n} \in A: r$. Let $P$ be any MPD of $A: r$. Then $P \supset P_{n}$ for some $n$; so if $P \neq P_{n}$, then $A: r \notin P_{n}$ and hence $x_{n} \in A: r \subset P$. Thus, $P=\left(x_{1}, x_{2}, \ldots\right)$ if $P \neq P_{n}$. But $r \notin A$ implies $r \notin P_{j}$ for some $j$; so $A: r \subset P_{j}<P$. Thus, $P$ is not an MPD of $A: r$ if $P \neq P_{n}$; so $\left\{P_{n}\right\}$ is exactly the set of $\mathrm{B}_{\mathrm{w}}$-primes of $A$.

Now let us check that $\sqrt{ }(A: r), r \in R$, has a.c.c. $A: r \neq A$ implies $r \in P_{n}$ for some $n$. Hence $r$ has constant term equal to 0 . If $r$ involves only the variables $x_{1}, \ldots, x_{m}$, then $x_{i} \in A: r$ for all $i>m$. Thus, the MPD's of $A: r$ can only come from among the set $P_{1}, \ldots, P_{m}$. Hence any proper ideal of the form $\sqrt{ }(A: r), r \in R$, is either $A$ or a finite intersection of ideals from $\left\{P_{n}\right\}$.

4. Generalizations of 1.2. Since Proposition 1.2 is crucial for the main result of $\$ 1$, it is perhaps of interest to examine the hypothesis further. Let us first observe 
that the proof remains valid if we merely assume that $R^{\prime}$ is a generalized quotient ring of $R$, in the sense of [1], i.e. there exists a homomorphism $\varphi: R \rightarrow R^{\prime}$ which makes $R^{\prime}$ a flat $R$-module, and $R^{\prime}$ is contained in the total quotient ring of $\varphi(R)$. Let us see what happens if one tries to weaken this assumption further to merely: $R^{\prime}$ is an $R$-algebra which is flat as an $R$-module. We make the following assumptions throughout this section: $R^{\prime}$ is an $R$-algebra which is flat as an $R$-module, $A$ is an ideal of $R, A^{\prime}=A R^{\prime}, P$ is a prime ideal of $R$ containing $A, P^{\prime}=P R^{\prime} \neq R^{\prime}$. By replacing $R$ by the canonical homomorphic image of $R$ in $R^{\prime}$ we may also, without loss of generality, assume that $R \subset R^{\prime}$. (The above assumptions also imply $P^{\prime} \cap R=P$.)

If $P^{\prime}$ is a prime ideal, then 4.3 will show that $P$ is a $\mathrm{B}_{\mathrm{w}}$-prime of $A$ implies $P^{\prime}$ is a $\mathrm{B}_{\mathrm{w}}$-prime of $A^{\prime}$. However, it can happen that $P$ is a $\mathrm{B}_{\mathrm{w}}$-prime of $A$ and yet $P^{\prime}=P R^{\prime}$ is not even prime. For example, take $R$ to be a valuation ring with quotient field $K$ having the property that there exists an algebraic extension $K^{\prime}$ of $K$ such that the integral closure $R^{\prime}$ of $R$ in $K^{\prime}$ is not a valuation ring. Then $R^{\prime}$ has at least two primes lying over $P\left[17\right.$, Vol. 2, p. 27, Theorem 12], so $P^{\prime}=P R^{\prime}$ cannot be prime. Note that by [3a, p. 29, Proposition 3], a module is flat over a Bezout domain if and only if the module is torsion free; in particular, $R^{\prime}$ is flat over $R$.

We can, of course, avoid the above difficulty by merely looking at the MPD's of $P^{\prime}$. We observe first that flatness of $R^{\prime}$ over $R$ yields the following proposition.

4.1. Proposition. If $z(A)$ and $z\left(A^{\prime}\right)$ denote, respectively, the zero divisors of $R$ modulo $A$ and of $R^{\prime}$ modulo $A^{\prime}$, then $z\left(A^{\prime}\right) \cap R \subset z(A)$. If in addition $A^{\prime} \cap R=A$, then $z(A)=z\left(A^{\prime}\right) \cap R$.

Proof. If $x \in z\left(A^{\prime}\right) \cap R$, then $(A: x) R^{\prime}=A^{\prime}: x \neq A^{\prime}$. Hence $A: x \neq A$ and $x \in z(A)$. Conversely, if $x \in z(A)$ and $A^{\prime} \cap R=A$, then $A: x \neq A$ implies that $A^{\prime} \neq(A: x) R^{\prime}$ $=A^{\prime}: x$ so that $x \in z\left(A^{\prime}\right)$.

4.2. Corollary. If $A$ is $P$-primary then any associated prime of $A^{\prime}$ in $R^{\prime}$ (using any one of the definitions of associated primes) lies over $P$.

Proof. This is immediate from 4.1, for if $Q^{\prime}$ is an associated prime of $A^{\prime}$, then $Q^{\prime} \subset z\left(A^{\prime}\right)$.

In particular, 4.2 implies that any MPD of $P^{\prime}$ in $R^{\prime}$ lies over $P$. It follows that for an arbitrary ideal $A$ of $R$ any MPD of $A R^{\prime}=A^{\prime}$ lies over an MPD of $A$.

4.3. Corollary. If $P$ is a $B_{w}$-prime of $A$, then each $M P D$ of $P^{\prime}$ is a $B_{w}$-prime of $A^{\prime}$.

Proof. $P$ is an MPD of $A: x$ for some $x \in R$. $(A: x) R^{\prime}=A R^{\prime}: x$ by flatness; so if $Q^{\prime}$ is an MPD of $P^{\prime}$, then $Q^{\prime}$ lies over $P$, and hence $Q^{\prime}$ is an MPD of $A R^{\prime}: x$. Q.E.D. 
It can happen, however, in the case where $R^{\prime}$ is not a generalized quotient ring of $R$, that $P^{\prime}$ is a $\mathrm{B}_{\mathrm{w}}$-prime of $A^{\prime}$ and yet $P$ is not a $\mathrm{B}_{\mathrm{w}}$-prime of $A$. Our next example illustrates this. (Example 4.4 also provides a negative answer to the more general question raised by D. Lazard in [9a, p. 19, Remark 3.4].)

4.4. EXAMPLE. Let $R$ be a valuation ring having maximal ideal $P$ and a chain of primes $P_{1}<P_{2}<\cdots$ such that $\bigcup P_{i}=P$ (such a ring may be obtained by choosing the value group of $R$ to be $Z \oplus Z \oplus \cdots$ with lexicographic order; see, for example, [6, p. 248]). Let $A=a R$ for some $a \neq 0$ in $P$. Then $A: y$ is principal for each $y \in R$, so $P$ cannot be an MPD of $A: y$. Thus $P$ is not a $\mathrm{B}_{\mathrm{w}}$-prime of $A$. Let $x$ be an indeterminate over $R$ and consider the ring $R^{\prime}=R+P^{\prime}$, where $P^{\prime}=P R[x / a]$. Since $R^{\prime} \subset R[x / a]$, we see that $R^{\prime}$ is a torsion free and hence flat $R$-module. Note that $P^{\prime} \cap R=P$ and $R^{\prime} / P^{\prime} \cong R / P$. Hence $P^{\prime}$ is a maximal ideal of $R^{\prime}$. Moreover, $P^{\prime}=P R^{\prime}$. It is clear that $P R^{\prime} \subset P^{\prime}$, and to show that $P^{\prime} \subset P R^{\prime}$ it will suffice to show that $b(x / a)^{i} \in P R^{\prime}$ for any $b \in P$. Since $P$ is not principal there exist $b_{1}, b_{2} \in P$ such that $b=b_{1} b_{2}$. Hence $b(x / a)^{i}=b_{1} b_{2}(x / a)^{i}$, and $b_{1} b_{2}(x / a)^{i} \in P R^{\prime}$ since $b_{2}(x / a)^{i} \in P^{\prime} \subset R^{\prime}$. Finally we show that $P^{\prime}=a R^{\prime}: x$, and hence that $P^{\prime}$ is a B-prime (and a fortiori a $\mathrm{B}_{\mathrm{w}}$-prime) of $a R^{\prime}=A R^{\prime}$. That $P^{\prime} \subset a R^{\prime}: x$ follows from the fact that $P \subset a R^{\prime}: x$, i.e. $x / a \cdot P \subset R^{\prime}$. Since $P^{\prime}$ is maximal, to show $P^{\prime}=a R^{\prime}: x$, it suffices to observe that $x / a \notin R^{\prime}=R+P R[x / a]$, which is clear from the transcendence of $x / a$ over $R$.

Thus, in general, $P^{\prime}$ is a $\mathrm{B}_{\mathrm{w}}$-prime of $A^{\prime}$ does not imply $P$ is a $\mathrm{B}_{\mathrm{w}}$-prime of $A$. We can show, however, that $P$ must be an $\mathrm{N}$-prime of $A$.

4.5. Proposition. If $Q^{\prime}$ is a $B_{w}$-prime of $A^{\prime}$ and $Q^{\prime} \cap R=P$, then $P$ is an $N$-prime of $A$.

Proof. If $S=R \backslash P$, then $R_{S}^{\prime}$ is flat over $R_{S}$ [3a, p. 115, Proposition 13]; and by Proposition 1.2, $Q^{\prime} R^{\prime}{ }_{S}$ is a $\mathrm{B}_{\mathrm{w}}$-prime of $A^{\prime} R^{\prime}{ }_{S}=A R^{\prime}{ }_{S}$. Hence $Q^{\prime} R^{\prime}{ }_{S}$ is contained in $z\left(A R^{\prime}\right)$, the set of zero divisors of $R^{\prime}{ }_{S}$ modulo $A R^{\prime}{ }_{S}$. We have $P R_{S} \subset Q^{\prime} R^{\prime}{ }_{S}$, so Proposition 4.1 implies that $P R_{S}$ consists of zero divisors modulo $A R_{S}$. Since $P R_{S}$ is a maximal ideal, it follows that $P R_{S}$ is a maximal $\mathrm{N}$-prime of $A R_{S}$. Hence $P$ is an N-prime of $A$. Q.E.D.

We have not been able to determine whether $N$-primes of $R^{\prime}$ necessarily contract to $\mathrm{N}$-primes of $R$ (even in the case where $R$ is an integral domain and $R^{\prime}$ is contained in the quotient field of $R$ ).

QUESTION. If $Q^{\prime}$ is an N-prime of $A^{\prime}$ must it follow that $Q=Q^{\prime} \cap R$ is an N-prime of $A$ ?

A difficulty in considering this question is that $\mathrm{N}$-primes are not preserved by localization, i.e. $Q$ is an N-prime of $A$ does not imply $Q R_{Q}$ is an N-prime of $A R_{Q}$. (See [10] or [5].) In connection with the above question we can make the following observations: applying 4.1 we see that $Q \subset z(A)$. Hence when $Q$ is maximal we certainly have that $Q$ is an $\mathrm{N}$-prime of $A$. Moreover, if there exists a multiplicative system $S^{\prime}$ of $R^{\prime}$ with $S^{\prime} \cap R=S$ such that $Q R_{S}$ is maximal and $Q^{\prime} R^{\prime}{ }_{S^{\prime}}$ is an Nprime of $A R_{S^{\prime}}^{\prime}$, then $Q R_{S}$ is an N-prime of $A R_{S}$ and hence $Q$ is an N-prime of $A$. 


\title{
REFERENCES
}

1. a. T. Akiba, Remarks on generalized rings of quotients, Proc. Japan Acad. 40 (1964), 801-806. MR 31 \#4807.

b. - - Remarks on generalized rings of quotients. II, J. Math. Kyoto Univ. 5 (1965), 39-44. MR 34 \#185.

2. J. T. Arnold and R. Gilmer, Idempotent ideals and unions of nets of Prüfer domains, J. Sci. Hiroshima Univ. Ser. A-I Math. 31 (1967), 131-145. MR 37 \#2741.

3. a. N. Bourbaki, Algèbre commutative. Chaps. 1, 2, Actualités Sci. Indust., no. 1290, Hermann, Paris, 1961. MR 36 \#146.

b. ——, Algèbre commutative. Chaps. 3, 4, Actualités Sci. Indust., no. 1293, Hermann, Paris, 1961. MR 30 \#2027.

c. - Algèbre commutative. Chaps. 5, 6, Actualités Sci. Indust., no. 1308, Hermann, Paris, 1964. MR 33 \#2660.

4. P. Eakin, The converse to a well known theorem on noetherian rings, Math. Ann. 177 (1968), 278-282. MR 37 \#1360.

5. R. Gilmer, A counterexample to two conjectures in ideal theory, Amer. Math. Monthly 74 (1967), 195-197.

6. R. Gilmer and J. Ohm, Primary ideals and valuation ideals, Trans. Amer. Math. Soc. 117 (1965), 237-250. MR 30 \#114.

7. W. Heinzer, J-noetherian integral domains with 1 in the stable range, Proc. Amer. Math. Soc. 19 (1968), 1369-1372. MR 38 \#145.

8. P. Jaffard, Les systèmes d'ideaux, Travaux et Recherches Mathématiques, IV, Dunod, Paris, 1960. MR 22 \#5628.

9. a. D. Lazard, Autour de la platitude, Thesis, Paris, 1968.

b. —_, Autour de la platitude, Bull. Soc. Math. France 97 (1969), 81-128.

10. M. Nagata, Some remarks on prime divisors, Mem. Coll. Sci. Univ. Kyoto Ser. A Math. 33 (1960/61), 297-299. MR 22 \#9518.

11. - A type of subrings of a noetherian ring, J. Math. Kyoto Univ. 8 (1968), 465-467. MR 38 \#4460.

12. - Local rings, Interscience Tracts in Pure and Appl. Math., no. 13, Interscience, New York, 1962. MR 27 \#5790.

13. N. Nakano, Idealtheorie in einem speziellen unendlichen algebraischen Zahlkörper, J. Sci. Hiroshima Univ. Ser. A 16 (1953), 425-439. MR 15, 510.

14. J. Ohm, Semi-valuations and groups of divisibility, Canad. J. Math. 21 (1969), 576-591. MR 39 \#4146.

15. J. Ohm and R. Pendleton, Rings with noetherian spectrum, Duke Math. J. 35 (1968), 631-639; Addendum, 875. MR 37 \#5201.

16. a. D. Underwood, Ideal theory in non-noetherian rings, Thesis, University of Wisconsin, Madison, Wis., 1968.

b. - On some uniqueness questions in primary representations of ideals, J. Math. Kyoto Univ. 9 (1969), 69-94. MR 40 \#134.

17. O. Zariski and P. Samuel, Commutative algebra. Vols. 1, 2, University Series in Higher Math., Van Nostrand, Princeton, N. J., 1958, 1960. MR 19, 833; MR 22 \#11006.

\author{
Purdue University, \\ LAFAYETTE, INDIANA 47907 \\ LOUISIANA STATE UNIVERSITY, \\ Baton Rouge, Louisiana 70803
}

